\title{
Insulin exposure mitigates the increase of arterial stiffness in patients with type 2 diabetes and albuminuria: an exploratory analysis
}

\author{
Daniel Gordin ${ }^{1,2,3} \cdot$ Markku Saraheimo $^{1,2,3} \cdot$ Jaana Tuomikangas ${ }^{1,2,3} \cdot$ Aino Soro-Paavonen $^{1,2,3} \cdot$ Carol Forsblom $^{1,2,3}$. \\ Karri Paavonen ${ }^{4}$. Birgit Steckel-Hamann ${ }^{5}$. Valma Harjutsalo ${ }^{1,2,3,6}$. Loizos Nicolaou . Imre Pavo $^{8}$. \\ Veikko Koivisto ${ }^{9}$. Per-Henrik Groop ${ }^{1,2,3,10}$
}

Received: 26 October 2018 / Accepted: 16 April 2019 / Published online: 22 May 2019

(c) The Author(s) 2019

\begin{abstract}
Aims Insulin possesses both vasodilatory and sympathomimetic activities. The aim was to examine the relationship between changes in insulin exposure and arterial stiffness in type 2 diabetes (T2D).

Methods Patients with T2D with $(n=22)$ or without $(n=24)$ albuminuria, and non-diabetic controls $(n=25)$ were randomized to a crossover study having a breakfast with or without pre-meal rapid-acting insulin. Pulse wave velocity (PWV) was measured at $30 \mathrm{~min}$ before and at 60 -min intervals up to $240 \mathrm{~min}$ after the breakfast.

Results At baseline, both postprandial aortic $(p=0.022)$ and brachial $(p=0.011)$ PWV were higher in individuals with T2D than in healthy controls irrespective of the presence of albuminuria. In patients with albuminuria, weight-adjusted insulin dose correlated inversely with the excursion of the aortic $(r=-0.412, p=0.006)$ and brachial $(r=-0.372 ; p=0.014)$ PWV. Similarly, circulating endogenous insulin concentrations correlated inversely with the aortic $(r=-0.347, p=0.026)$ and brachial $(r=-0.622, p=<0.001)$ PWV. No correlations between insulin and PWV were observed in patients without albuminuria or in healthy controls.

Conclusions The inverse correlation between insulin and PWV in T2D with albuminuria may reflect a vasorelaxing effect of insulin.
\end{abstract}

Clinical trial registration number The study was registered (clinicaltrials.gov) with the identifier of NCT01159938.

Keywords Type 2 Diabetes $\cdot$ Pulse wave velocity $\cdot$ Arterial stiffness $\cdot$ Insulin resistance $\cdot$ Albuminuria $\cdot$ Diabetic nephropathy $\cdot$ Diabetic kidney disease

\section{Introduction}

The role of insulin in enhancing peripheral blood flow as well as increasing arterial diameter in large arteries is well established [1-3]. The vasodilatory effect of insulin can be

Managed by Giuseppe Pugliese.

Per-Henrik Groop

per-henrik.groop@helsinki.fi

5 Eli Lilly \& Co Export SA, Geneva, Switzerland

6 Chronic Disease Prevention Unit, National Institute for Health and Welfare, Helsinki, Finland

1 Folkhälsan Institute of Genetics, Folkhälsan Research Center, Biomedicum Helsinki (C318b), Haartmaninkatu 8, 00290 Helsinki, Finland

2 Abdominal Center Nephrology, University of Helsinki and Helsinki University Hospital, Helsinki, Finland

3 Research Programs Unit, Diabetes and Obesity, University of Helsinki, Helsinki, Finland

ClinBAY, Genappe, Belgium

8 Eli Lilly \& Co, Vienna, Austria

9 Eli Lilly \& Co, Helsinki, Finland

10 Department of Diabetes, Central Clinical School, Monash University, Melbourne, VIC, Australia

4 Department of Medicine, University of Helsinki and Helsinki University Hospital, Helsinki, Finland 
blocked by inhibiting nitric oxide synthesis highlighting the endothelial effects of insulin [4]. It is not known if the vasodilatory effect of insulin is preserved in patients with diabetic complications. An open but important question is whether albuminuria, which is often related to autonomous neuropathy and endothelial dysfunction, influences the effect of insulin on blood flow and/or arterial stiffness [5-8]. In the absence of autonomic neuropathy, there is synchrony between vasodilatory and vasoconstrictive effects of insulin in maintaining appropriate blood flow. We have previously shown that postprandial hyperglycemia increases brachial pulse wave velocity (PWV) in albuminuric but not in normoalbuminuric patients with type 2 diabetes (T2D) [9]. Now we analyzed whether there is any association between postprandial hyperinsulinemia and the stiffness of large (aortic) and middle-sized (brachial) arteries in individuals with T2D, and if so, are there differences between T2D patients with or without albuminuria.

\section{Subjects, study design and methods}

\section{Subjects}

Twenty-two patients with type 2 diabetes with and 24 patients without albuminuria, and 25 non-diabetic control subjects were studied after a high-carbohydrate meal. Eligible individuals were male between 45 and 70 years of age, had not smoked in the $12 \mathrm{~h}$ before the visits, and had not experienced cardiovascular event such as coronary heart disease, stroke or peripheral vascular disease. All 24 patients with albuminuria, 21/22 patients without albuminuria and 5/20 control subjects were on antihypertensive medication. ACE inhibitors/ATII blockers were used by $5 / 10$ patients, with albuminuria, $2 / 5$ patients without albuminuria, and 6/1 control subject. Cholesterol-lowering agents (mostly statins) were used by $73 \%$ of patients with albuminuria, $92 \%$ of patients without albuminuria and $8 \%$ of the control subjects as described previously [9]. Their clinical characteristics are shown in Table 1. The study protocol is in accordance with the Declaration of Helsinki as revised in 2000, and approved by the local ethics committee. Written informed consent was obtained from each patient.

\section{Study design}

The study was performed over two single-day clinical visits. Patients (stratified by the presence or absence of albuminuria) were randomized to two treatment schedules: either insulin lispro was or was not injected subcutaneously before a standard breakfast. The patients injected their basal insulin (insulin glargine or detemir) as usual before the study. Healthy individuals were studied only once. Blood samples were drawn and PWV was measured $30 \mathrm{~min}$ before and $60,120,180$, and $240 \mathrm{~min}$ after the breakfast meal. The design and the composition of the meals have already been published in the previous paper [9]. In brief, this study was done in three single-day visits. During visit 1 , the patients with diabetes were randomized blindly (albuminuria vs normal UAER) to have insulin lispro subcutaneously before

Table 1 Subject characteristics

\begin{tabular}{llll}
\hline & $\begin{array}{l}\text { Non- } \\
\text { diabetic } \\
\text { controls } \\
N=25\end{array}$ & Patients with albuminuria & Patients with normal UAER \\
& $N=22$ & $N=24$ \\
\hline Age (years) & $59 \pm 7$ & $61 \pm 5(0.148)$ & $64 \pm 5(0.004)$ \\
BMI (kg/m $\left.{ }^{2}\right)$ & $27 \pm 3$ & $34 \pm 5(<0.001)$ & $32 \pm 6(<0.001)$ \\
Disease duration (years) & $\mathrm{NA}^{\mathrm{a}}$ & $12 \pm 4$ & $16 \pm 8$ \\
UAER (ug/min) & $\mathrm{NA}^{\mathrm{a}}$ & $273 \pm 476$ & $5 \pm 5$ \\
HbA $(\%)(\mathrm{mmol} / \mathrm{mol})$ & $5.4 \pm 0.3$ & $7.8 \pm 1.3(<0.001)$ & $7.4 \pm 1.1(<0.001)$ \\
& $35 \pm 1.5$ & $62 \pm 6.5$ & $57 \pm 5.5$ \\
Total cholesterol (mmol/l) & $5.3 \pm 0.8$ & $4.1 \pm 0.7(<0.001)$ & $4.3 \pm 0.9(<0.001)$ \\
Triglycerides (mmol/l) & $1.3 \pm 0.5$ & $1.5 \pm 0.8(0.554)$ & $1.6 \pm 1.5(0.401)$ \\
Insulin dose (U) & & & $11 \pm 6$ \\
Lispro & $\mathrm{NA}$ & $13 \pm 14$ & $61 \pm 49$ \\
Basal insulin. high PP glucose & $\mathrm{NA}$ & $80 \pm 53$ & $61 \pm 50$ \\
Basal insulin. low PP glucose & $\mathrm{NA}$ & $78 \pm 53$ & \\
\hline
\end{tabular}

Values inside the brackets illustrate $p$ values for pairwise comparison to healthy volunteers

$B M I$ body mass index, UAER urinary albumin excretion

${ }^{\mathrm{a}}$ Not analyzed for statistical difference 
breakfast meal either during visit 2 or visit 3 . The breakfast included $500 \mathrm{kcal}, 60 \%$ carbohydrate, $20 \%$ protein and $20 \%$ fat. The day insulin lispro was injected, the rise in glucose was less (low-glucose study) than on the day lispro was not injected (high-glucose study). Insulin dosing was based on the patient's normal morning insulin dose and the energy content of the meal. If the study breakfast was larger than the patient's normal breakfast, insulin lispro dose was increased by $20-50 \%$ at the discretion of the investigator.

\section{Methods}

To measure the arterial stiffness in the large (carotid-femoral or aortic) and intermediate-sized (carotid-radial or brachial) arteries, PWV pressure waveforms were recorded sequentially at the carotid, femoral, and radial arteries with a high-fidelity micromanometer (SPC-301; Millar Instruments: SphygmoCor, Sydney Australia). With a simultaneous EKG recording of the $\mathrm{R}$-wave as reference frame, the software calculated the PWV [10].

Blood glucose was measured by the glucose oxidase method using a HemoCue $201 \mathrm{~m}$ (Hemocue, Ängelholm, Sweden). Insulin was quantitated with a immunoelectrochemiluminometric assay on the Modular Analytics E170 analyzer (Roche Diagnostics, Mannheim, Germany). The detection limit of the assay is $0.2 \mathrm{mU} / \mathrm{L}$. Intra-assay coefficient of variation is $<2 \%$ and inter-assay variation $<5 \%$ at $7-400 \mathrm{mU} / \mathrm{L}$. Cross-reaction with insulin analogues lispro, aspart, glargine, detemir and glulisine is $<0.1 \%[11,12]$. The assay is calibrated against WHO 1st IRP NIBSC 66/304.

\section{Statistical analyses}

Pearson correlation coefficient was used to summarize the association between insulin and PWV. The observed area under the curve (AUC) $\left(\mathrm{AUC}_{0-4 \mathrm{~h}}\right)$ not adjusted for baseline values as in our previous paper [9]) was calculated for PWV and the insulin concentrations. The least square (LS) means, obtained from an ANCOVA model (including age and BMI as covariates and the subject group as factor) were summarized separately by subject group and glycemic condition (i.e., low or high). The LS means were compared between the subject groups and the significance was assessed at the two-sided 5\% level. Finally, to test whether insulin has a similar effect on PWV (similar slopes) in patients with and without albuminuria and in the control group, the interaction term between the groups and insulin as continuous measurement was incorporated in the model. While the individuals with T2D were studied during two periods (i.e., under high- and low-postprandial glucose conditions), the non-diabetic controls were studied only for one period. The model predicted mean data per time point for the control group were different between the two glycemic conditions due to the effect of adjusting for covariates.
Statistical analyses were performed using SAS 9.4 version (SAS Institute Inc, Cary, NC, USA).

\section{Results}

\section{Blood glucose}

In the two diabetes patient groups combined, the LS mean \pm SE glucose level was $6.8 \pm 0.2 \mathrm{mmol} / \mathrm{L}$ before breakfast. The peak postprandial rise in blood glucose after breakfast was lower with $(3.3 \pm 0.3 \mathrm{mmol} / \mathrm{L}, p=0.001)$ than without $(5.3 \pm 0.3 \mathrm{mmol} / \mathrm{L})$ pre-meal lispro injection $(p=0.001)$. While the glucose levels were significantly higher throughout the study in both diabetes groups as compared to healthy controls $(p<0.01-0.001)$, there were no differences in postprandial glucose levels between patients with or without albuminuria [9].

\section{Insulin dosages and concentrations}

There was no significant difference in the mean doses $(\mathrm{U} \pm \mathrm{SD})$ of insulin lispro and basal insulin (glargine or levemir) in patients with albuminuria (lispro $13.4 \pm 13.7+$ basal $80.1 \pm 53.3$ vs $77.7 \pm 53.4$ basal only in low- and high-glucose studies, respectively) compared to patients without albuminuria (lispro $11.2 \pm 6.1+$ basal $60.8 \pm 49.5$ vs $61.3 \pm 50.0$ basal only). Neither was there any significant difference in the LS mean AUC $( \pm$ SE) of serum insulin concentrations in the high- and low-glucose studies, respectively, in patients with $(96.4 \pm 18.6$ and $84.1 \pm 18.3 \mathrm{mU} * \mathrm{~h} / \mathrm{L})$ or without albuminuria $(119.2 \pm 17.2$ and $102.1 \pm 17.4 \mathrm{mU} * \mathrm{~h} / \mathrm{L}$ ), or between the diabetes patient groups. In healthy individuals, the mean serum insulin AUC was $109.7 \pm 19.7 \mathrm{mU} * \mathrm{~h} / \mathrm{L}$, not significantly different from the two diabetes groups. There was no significant difference between the diabetic groups in insulin AUC as such, or when AUC was adjusted by weight.

\section{PWV}

At baseline, the LS mean $( \pm S E)$ of AUC PWV in the aorta was higher in patients with $(42.3 \pm 2.4$ and $41.9 \pm 2.27 \mathrm{~m} * \mathrm{~h} / \mathrm{s})$ and without albuminuria $(41.2 \pm 2.3$ and $41.6 \pm 2.3 \mathrm{~m} * \mathrm{~h} / \mathrm{s}$ ) as compared to healthy controls $(34.3 \pm 2.3$ and $36.0 \pm 2.3 \mathrm{~m} * \mathrm{~h} / \mathrm{s}, p=0.022$ and 0.072$)$, while there was no difference between the two diabetes subgroups. Similarly, the LS mean $( \pm \mathrm{SE})$ of AUC brachial PWV was higher in patients with $(32.1 \pm 1.1$ and $32.9 \pm 1.2 \mathrm{~m} * \mathrm{~h} / \mathrm{s})$ and without albuminuria $(30.6 \pm 1.0$ and $31.2 \pm 1.2 \mathrm{~m} * \mathrm{~h} / \mathrm{s}$ ) as compared to healthy controls $(27.7 \pm 1.1$ and $27.6 \pm 1.3 \mathrm{~m} * \mathrm{~h} / \mathrm{s}, p=0.011$ and 0.009$)$, 
while there was no difference between the two diabetes subgroups.

\section{Correlation between insulin and PWV}

In patients with albuminuria, there was a significant inverse correlation between total insulin dose/weight and AUC aortic (Fig. 1) and brachial PWV (Fig. 2). Similarly, there was an inverse relationship between AUC of endogenous serum insulin concentration and AUC aortic PWV (Fig. 3) and brachial PWV (Fig. 4). When glucose was included in these correlations (insulin dose/weight/AUC glucose vs AUC PWV), the significance remained in the albuminuric patients for aortic $(r=-0.383, p<0.011)$ and brachial PWV ( $r=-0.372, p<0.014)$. Equally, the correlations after adjustment for serum glucose between the AUC serum insulin concentration/weight/AUC glucose and the AUC PWV were significant in the albuminuric patients for aortic $(r=-0.381, p<0.014)$ and brachial PWV $(r=-0.571$, $p<0.001)$. Inclusion of total cholesterol and eGFR in the model on top of the previous covariates did not influence the associations between insulin and arterial stiffness further strengthening our results (data not shown). Notably, in patients without albuminuria or in healthy controls no such correlations between insulin and PWV were observed. However, the slopes were significantly different between patients with and without albuminuria only in case of brachial PWV, as the interaction term between albuminuria groups and insulin was significant ( $p=0.002$ for both AUC insulin concentration, and total insulin dose). No interactions were observed between controls and either of the diabetes groups. The data were further separately analyzed for high- or low-postprandial glucose conditions. The associations between brachial PWV and insulin dose/weight during high- and low-postprandial conditions were $r=-0.543$, $p=0.013$ and $-0.659, p=0.001$, respectively. The associations between aortic PWV and insulin dose/weight during
Fig. 1 Correlation between AUC aortic PWV and total insulin dose/weight in patients with type 2 diabetes with albuminuria. For each patient, a study with and without pre-meal lispro is included
Fig. 2 Correlation between AUC brachial PWV and total insulin dose/weight in patients with type 2 diabetes with albuminuria. For each patient, a study with and without pre-meal lispro is included
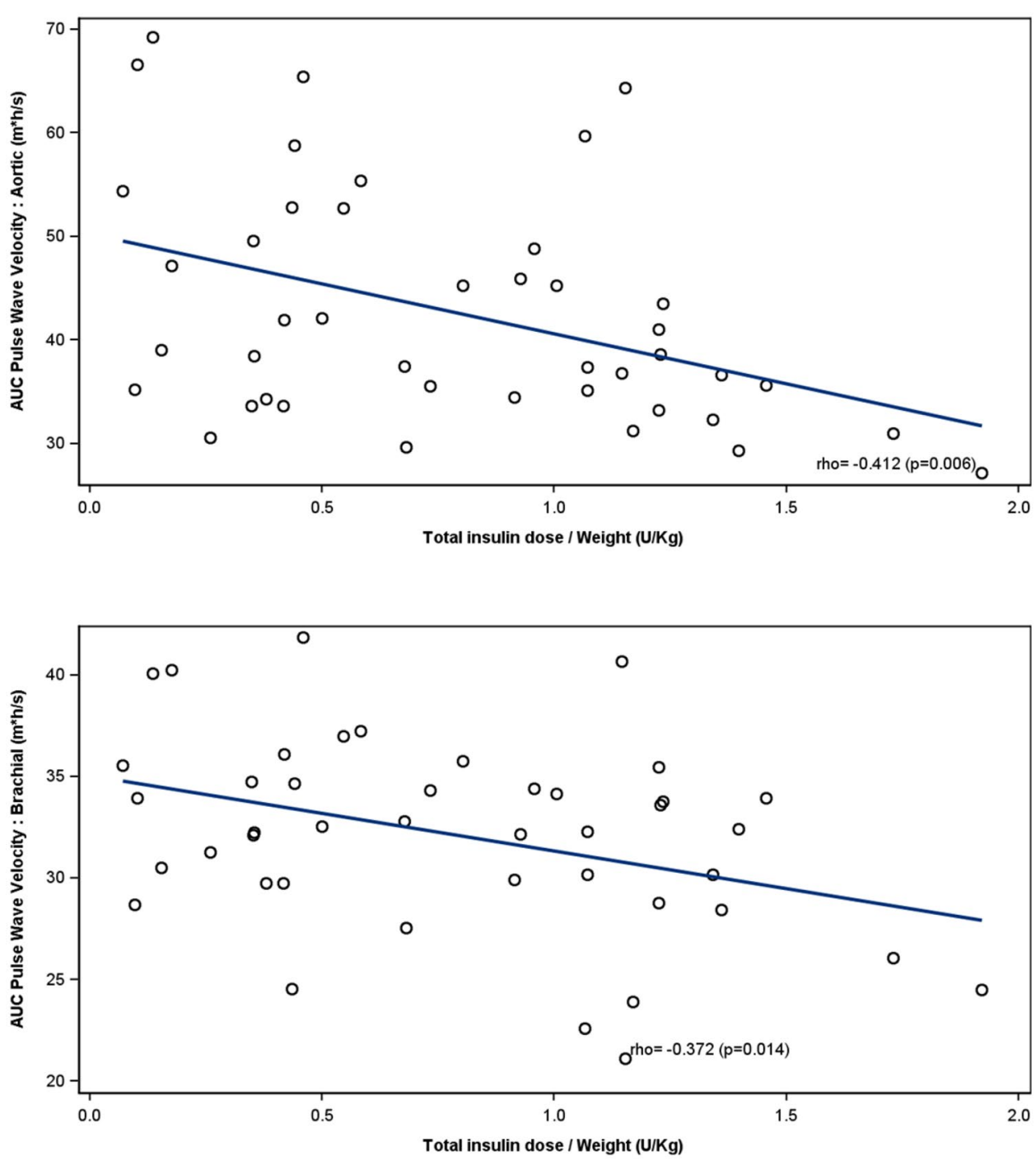

AUC aortic PWV and AUC of endogenous serum insulin concentration in patients with type 2 diabetes with albuminuria. For each patient, a study with and without pre-meal lispro is included
Fig. 3 Correlation between

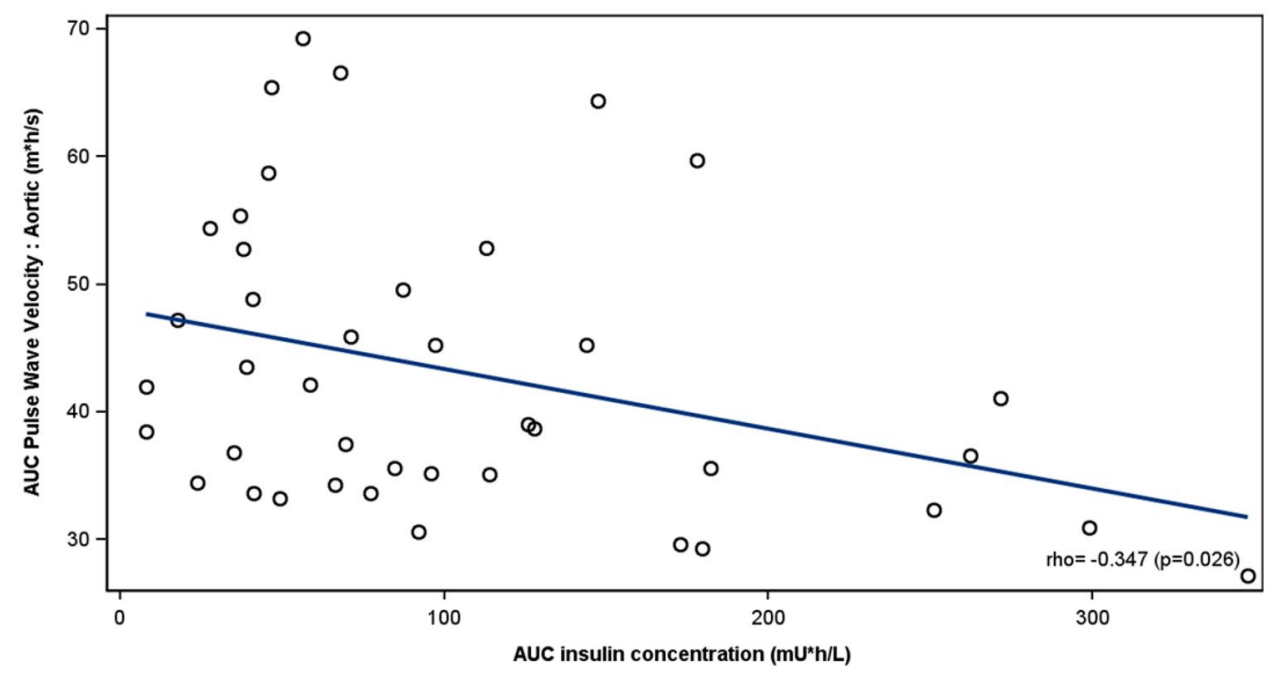

Fig. 4 Correlation between AUC brachial PWV and AUC of endogenous serum insulin concentration in patients with type 2 diabetes with albuminuria. For each patient, a study with and without pre-meal lispro is included

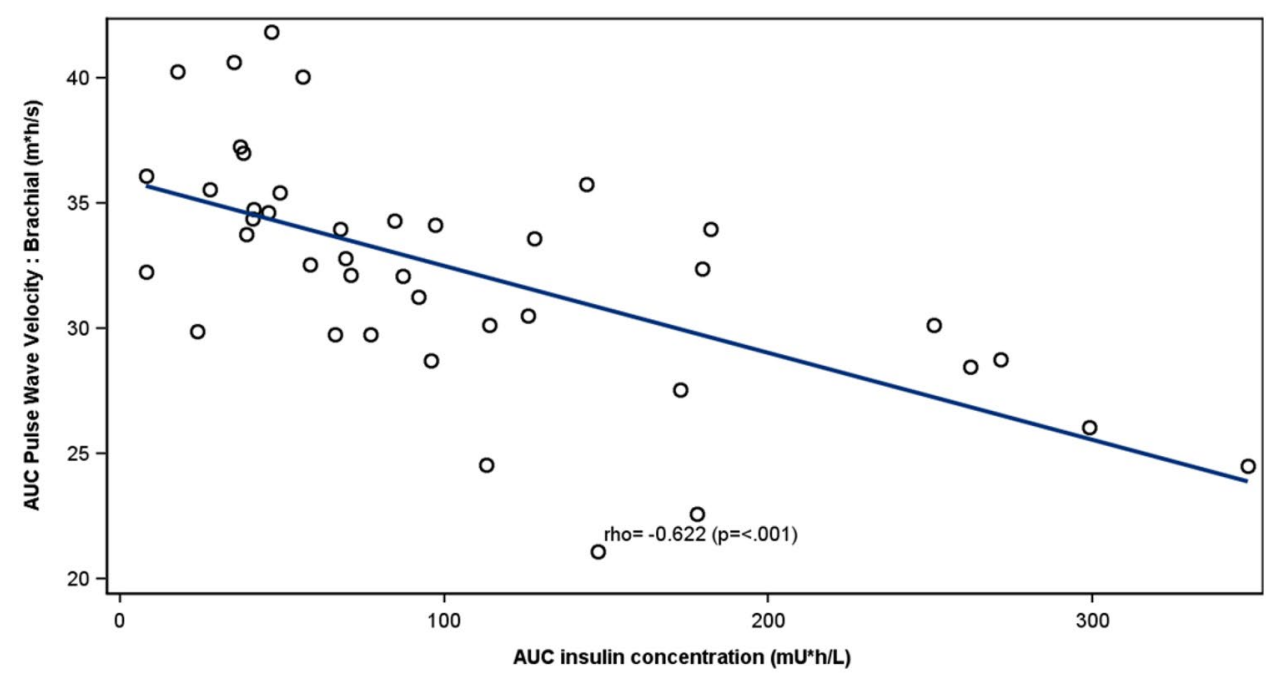

high -and low-postprandial conditions were $0.574, p=0.008$ and $-0.442, p=0.039$, respectively.

\section{Discussion}

We observed an inverse correlation between insulin and PWV in the T2D patients with but not without albuminuria or in healthy controls. Microalbuminuria is associated with autonomic neuropathy $[6,7]$ and endothelial dysfunction [8]. Since insulin plays a key role in the regulation of arterial blood flow, tone and endothelial function [13], we examined whether albuminuria influences the association between insulin availability and postprandial PWV in large arteries.

The correlation between postprandial arterial stiffness $(\mathrm{PWV})$ and insulin under hyperglycemic conditions in patients with T2D remained statistically significant, when insulin dose/weight or serum insulin concentration was adjusted for the plasma glucose concentration suggesting that the association is independent of the glycemia and may reflect a direct insulin-medicated effect. We had a specific assay for endogenous insulin, but did not have a specific assay for lispro or insulin glargine or detemir. For this reason, we used the insulin dose as a second measure of insulin availability. There was an inverse correlation between PWV and with both endogenous insulin as well as insulin dose, supporting our hypothesis of a correlation between insulin and PWV. Surprisingly, these correlations were neither present in T2D patients without albuminuria nor in healthy individuals. The significant difference between the albuminuric and the non-albuminuric patients regarding the correlation coefficients trends of insulin vs PWV further supports that insulin has a different effect on PWV in these two patient groups. A potential role of insulin is further supported by the fact that there was no difference between the groups regarding the prevalence of cardiovascular disease, cholesterol-lowering or antihypertensive medications. In this particular study, the original aim was to examine 
the effect of postprandial hyperglycemia on PWV. For this reason, we selected patients with type 2 diabetes, who were using basal-bolus regimens so that one study can be done with and another without pre-meal short-acting insulin. This design served well to examine a potential relationship of PWV and postprandial insulin, endogenous or exogenous. We could not observe differences in established biomarkers reflecting inflammation, endothelial function or advanced glycated end-products (sCRP, sICAM-1, sVCAM-1, ADMA, sRAGE) between the groups [9].

The finding may be explained by insulin's dual role in the vasculature. Although insulin is a vasodilator, concomitantly it can also constrict the arteries through the autonomic nervous system [5]. The balance and the shift in the balance of the sympatico-excitatory and the vasodilator effects of insulin can occur already at modestly elevated plasma insulin levels [9], as our patients had. One could anticipate that patients with T2D and albuminuria have advanced autonomic neuropathy $[6,7]$, which can diminish the vasoconstrictive effects of insulin. As a result, the vasodilatory effects of insulin were dominant in the current setting resulting in a reduced PWV. Importantly, we can only speculate on these mechanisms as we lack direct measures of autonomic neuropathy in this study. Another contributing factor could be among patients with albuminuria a higher sensitivity to insulin and its vasodilatory effects as compared to patients without albuminuria. While patients were using exogenous insulin, their serum endogenous insulin concentrations were similar to healthy individuals, probably reflecting insulin resistance.

This study does not go without limitations. The study groups were relatively small and the correlations might be influenced by a few extreme observations. We have, therefore, presented the data in scatter plots showing the response of individual subjects. Regarding the insulin exposure, there were no differences in insulin doses or areas under the curves for endogenous insulin between albuminuric and non-albuminuric patients. Thus, differences in insulin exposure cannot explain the difference in the relationship of PWV and insulin in the two patient groups. We used the insulin dose as an approximate of total exogenous insulin exposure which may not be an exact measure. This approach was, however, consistently used throughout the study. We cannot exclude the fact that albuminuria would have been masked by the use of RAAS inhibitors in this real-life study. We did not have any specific measures of autonomic neuropathy, but it is well established that patients with albuminuria have a more advanced autonomic neuropathy than patients without albuminuria [6].

In conclusion, we observed an inverse correlation between insulin and arterial stiffness in large (aortic) and middle-sized (brachial) arteries measured as PWV in individuals with $\mathrm{T} 2 \mathrm{D}$ and albuminuria. This was not observed in patients with T2D but without albuminuria or in non-diabetic individuals. Potentially, insulin counteracts the vasoconstriction caused by endothelial dysfunction and increased sympathetic activity known to be present in albuminuric patients with T2D. Interestingly, we observed a positive correlation between PWV and postprandial serum glucose in the same patients, and only again in the patients with albuminuria [9]. Thus, it is possible that in albuminuric patients, the arteries are sensitive to acute stiffening by glucose, and to relaxation by insulin.

Acknowledgements Open access funding provided by University of Helsinki including Helsinki University Central Hospital. Technical support of Maikki Parkkonen (laboratory manager at the Folkhälsan Institute of Genetics), and serum insulin determinations by Henrik Alfthan, Helsinki University Hospital, is acknowledged. This work was supported by Eli Lilly \& Co. The research received support from the Innovative Medicines Initiative Joint Undertaking, under Grant 115006, the resources of which are composed of a financial contribution from the Seventh Framework Programme from the European Union (Grant FP7/2007-2013) and the European Federation of Pharmaceutical Industries and Associations in kind contribution.

Author contributions DG, P-HG, VK, JT, and BS-H contributed substantially to the conception and/or design of the study. MS, AS-P, DG, $\mathrm{CF}, \mathrm{KP}, \mathrm{JT}, \mathrm{VH}$ and IP contributed substantially to the acquisition and/or interpretation of the data. $\mathrm{VH}$ and $\mathrm{LN}$ were responsible for the statistical analysis of the data. All authors reviewed and appraised the manuscript and approved the final version of the manuscript for submission. P-HG is the guarantor of this work, and, as such, had full access to all data in the study and takes the responsibility for the integrity of the data and accuracy of the data analysis

\section{Compliance with ethical standards}

Conflict of interest IP and BS-H are employees and VK is a contractor of Eli Lilly \& Co, the manufacturer of Humalog. P-HG has received lecture honoraria from AstraZeneca, Boehringer Ingelheim, Eli Lilly, Elo Water, Genzyme, Medscape, MSD, Novartis, Novo Nordisk, and Sanofi, and is on the advisory boards of AbbVie, AstraZeneca, Boehringer Ingelheim, Eli Lilly, Janssen, MSD, Novartis, Novo Nordisk, and Sanofi. P-HG has received a grant from Eli Lilly for this study as well as another investigator-initiated grant from Roche. The other authors have nothing to disclose.

Statement of human and animal rights The study protocol is in accordance with the Declaration of Helsinki as revised in 2000 and was approved by the local ethics committee in each study center.

Informed consent Written informed consent was obtained from each patient.

Open Access This article is distributed under the terms of the Creative Commons Attribution 4.0 International License (http://creativeco mmons.org/licenses/by/4.0/), which permits unrestricted use, distribution, and reproduction in any medium, provided you give appropriate credit to the original author(s) and the source, provide a link to the Creative Commons license, and indicate if changes were made. 


\section{References}

1. Yki-Järvinen H, Utriainen T (1998) Insulin-induced vasodilatation: physiology or pharmacology. Diabetologia 41:369-379

2. Lambert J, Smulders RA, Aarsen M, Gallay FP, Stehouser CDA (1997) The acute effect of hyperglycemia on vessel wall properties. Scand J Clin Invest. 57:409-414

3. Westerbacka J, Wilkinson I, Cockcroft J, Utriainen T, Vehkavaara S, Yki-Järvinen H (1999) Diminished wave reflection in the aorta. A novel physiological action of insulin on large blood vessels. Hypertension 33:1118-1122

4. Steinberg HO, Brechtel G, Johnson A, Finsberg N, Baron AD (1994) Insulin mediated skeletal muscle vasodilatation is nitric oxide dependent: a novel action of insulin to increase nitric oxide release. J Clin Invest 94:1172-1179

5. Deckert T, Feldt-Rasmussen B, Borch-Johnsen K, Jensen T, Kofoed-Eneveldsen A (1989) Albuminuria reflects widespread vascular damage: the Steno hypothesis. Diabetologia 32:219-226

6. Moran A, Palmas W, Field L et al (2004) Cardiovascular autonomic neuropathy is associated with microalbuminuria in older patients with type 2 diabetes. Diabetes Care 27:972-977

7. Wieland RG (1996) Association of cardiac autonomic neuropathy and microalbuminuria in non-insulin-dependent diabetes mellitus. Endocr. Pract 2:98-99

8. Dogra G, Rich L, Stanton K, Watts GF (2001) Endothelium-dependent and independent vasodilatation studied at normoglycemia in type 1 diabetes mellitus with and without microalbuminuria. Diabetologia 44:593-601

9. Gordin D, Saraheimo M, Tuomikangas J et al (2016) Influence of postprandial hyperglycemic conditions on arterial stiffness in patients with type 2 diabetes. J Clin Endocr Metab 101:1134-1143

10. Wilkinson IB, Fuchs SA, Jansen IM et al (1998) Reproducibility of pulse wave velocity and augmentation index measured by pulse wave analysis. J Hypertens 16:2079-2084

11. Owen WE, Roberts WL (2004) Cross reactivity of three recombinant insulin analogues with five commercial insulin immunoassays. Clin Chem 50:257-259

12. Sapin R (2003) Insulin assays: previously known and new analytical features. Clin Lab 49:113-121

13. Park K, Li Q, Evcimen ND et al (2017) Exogenous insulin infusion can decrease atherosclerosis in diabetic rodents by improving lipids, inflammation, and endothelial function. Arterioscler Thromb Vasc Biol 1:2. https://doi.org/10.1161/ATVBA HA.117.310291

Publisher's Note Springer Nature remains neutral with regard to jurisdictional claims in published maps and institutional affiliations. 